\title{
Simultaneous Topography-guided Surface Ablation with Collagen Cross-linking for Keratoconus
}

\author{
${ }^{1}$ Lily Karmona, ${ }^{2}$ Tzahi Sela, ${ }^{3} \mathrm{Oz}$ Franco, ${ }^{4}$ Avi Shoshani, ${ }^{5}$ Gur Munzer, ${ }^{6,7}$ Igor Kaiserman
}

\begin{abstract}
Purpose: In this article, we present the results of eight eyes of patients who underwent combined same-day partial topographyguided surface ablation followed by accelerated collagen cross-linking $(\mathrm{CXL})$ procedure at Care-Vision Laser Centers to achieve stabilization of corneal ectasia and enhance visual rehabilitation in keratoconus.
\end{abstract}

Materials and methods: A retrospective nonrandomized review of 8 eyes of 6 patients who underwent the Athens Protocol for progressive keratoconus. Each patient underwent topography-guided ablation followed by CXL procedure.

Follow-up: Follow-up was done on day 1 , day 7 , and then at $1,3,6$, and 12 months.

Results: There was a rapid and significant improvement in uncorrected visual acuity and best-corrected visual acuity in $100 \%$ eyes, a reduction of keratometric values, and symmetry between vertical hemimeridians. Topographic evaluation showed a marked improvement in irregularity. There were no signs of keratoconic progression noted in any of the eyes on last follow-up. No adverse events were reported in any patient.

Conclusion: Simultaneous surface ablation $+\mathrm{CXL}$ seems to be a promising treatment capable of offering patients functional vision and halting progression of the disorder.

Precis: Simultaneous topography-guided custom ablation treatment and photorefractive keratectomy with $\mathrm{CXL}$ offers keratoconic patients intolerant to contact lenses both stabilization of the cornea and improved functional vision with spectacles correction.

Keywords: Athens protocol, Keratoconus, Topography-guided surface ablation.

How to cite this article: Karmona L, Sela T, Franco O, Shoshani A, Munzer G, Kaiserman I. Simultaneous Topography-guided Surface Ablation with Collagen Cross-linking for Keratoconus. Int J Kerat Ect Cor Dis 2016;5(2):71-76.

Source of support: Nil

Conflict of interest: None

\footnotetext{
${ }^{1}$ Tutor and Research Assistant Professor, ${ }^{2}$ Directors of Administrative, ${ }^{3,4}$ Associate/Assistant Directors of Administrative ${ }^{5}$ Director General, ${ }^{6,7}$ Professor

${ }^{1}$ Department of Ophthalmology, Wolfson Medical Center, Holon Israel

${ }^{2-6}$ Care-Vision Laser Centers, Tel-Aviv, Israel

${ }^{7}$ Department of Ophthalmology, Barzilai Medical Center Ashkelon, Israel

Corresponding Author: Lily Karmona, Tutor and Research Assistant Professor, Department of Ophthalmology, Wolfson Medical Center, Holon, Israel, Phone: +972036916371 +9720523260588, e-mail: llekarmona@yahoo.com
}

\section{INTRODUCTION}

Keratoconus is a bilateral, noninflammatory progressive ectatic disorder of the cornea characterized by progressive corneal thinning and bulging. The cornea assumes a conical shape due to its biomechanical instability, which leads to irregular astigmatism and decrease in visual acuity. ${ }^{1}$

Treatment options must improve two distinct parameters: Optical inefficiency of the irregular cornea and corneal biomechanical instability. The optical inefficiency can be corrected with spectacles and contact lenses in the initial stages of the disease process. However, with continued progression, several surgical options, such as intrastromal corneal ring segments, ${ }^{2}$ lamellar keratoplasty, or penetrating keratoplasty may be needed. Prior to the introduction of corneal collagen cross-linking (CXL), there was no treatment capable of correcting the biomechanical instability. However, recently surgeons have successfully used CXL to impede the progression of keratoconus.

Collagen cross-linking with the use of riboflavin (vitamin B2) and ultraviolet A (UVA) irradiation crosslinks the collagen fibers in the corneal stroma, thereby strengthening and biomechanically stabilizing the cornea. $^{3}$

Using CXL alone provided patients with stability; however, the optical inefficiency persists secondary to the irregular astigmatism. Therefore, visual rehabilitation is still necessary after CXL. ${ }^{4,5}$

The earlier treatment of corneas with keratoconus using excimer laser was difficult as well as considered inappropriate, as the laser treatment results in further thinning of the cornea, and possible destabilization of the corneal structure and worsening of ectasia.

Recently, improvements in laser technology have led to several options, such as topography-guided laser ablation, for dealing with irregular astigmatism. The concept of topography-guided custom ablation treatment (T-CAT) is to modify the cornea by treating anatomical rather than refractive changes and to improve the central corneal symmetry without attempting to correct other spherical or regular astigmatic, optical defects. Kanellopoulos et al have recently introduced the Athens Protocol, a novel combined approach of simultaneous topography-guided surface ablation with CXL. The topography-guided 
surface ablation normalizes the corneal surface by reducing irregular astigmatism and potentially the refractive error, and the CXL then halts progression of the disorder. ${ }^{6-8}$ Patients experience improved visual outcomes in addition to stabilization of the ectatic process. By not attempting to correct the whole optical defect of the eye, T-CAT can be kept to a small degree of ablation with the maximum depth of tissue loss typically being less than $50 \mu \mathrm{m}$. Therefore, T-CAT is a procedure of limited ablation of the cornea using excimer laser with the aim of regularizing the cornea, improving the quality of vision and possibly contact lens fit. The aim of this procedure was not to give a complete refractive correction. ${ }^{9}$ Topographyguided custom ablation treatment with photorefractive keratectomy (PRK), as opposed to T-CAT alone, can also correct the complete refractive error.

In this article, we present the results of this combined same-day partial topography-guided surface ablation followed by accelerated CXL procedure to achieve stabilization of corneal ectasia and enhance visual rehabilitation.

\section{MATERIALS AND METHODS}

This is a retrospective nonrandomized review of 8 eyes of 6 patients who underwent simultaneous topographyguided surface ablation with CXL for progressive keratoconus at Care-Vision Laser Centers.

Each patient underwent assessment of uncorrected visual acuity (UCVA) and best-corrected visual acuity (BCVA) on the Snellen's chart, manifest refraction, cycloplegic refraction, scotopic pupillometry, and slit-lamp examination of the anterior and posterior segments of the eye. Keratometry readings, pachymetry, and corneal topography were performed using Sirius Scheimpflug camera (Sirius, Florence, Italy). Corneal asphericity (Q value) was measured using the Topolyzer.

Inclusion criteria were mild-to-moderate grade keratoconus, thinnest pachymetry $>450 \mu \mathrm{m}$, poor contact lens fit, unhappy with glasses or contact lenses, no active allergic eye disease, no active ocular inflammation, and no central scarring of the cornea.

\section{SURGICAL PROCEDURE}

All procedures were performed by the same surgeon in two consecutive steps:

Step 1: Topography-guided surface ablation

After topical anesthesia with oxybuprocaine $0.4 \%$ eye drop, a 8-mm diameter zone of corneal epithelium is removed by phototherapeutic keratectomy, and afterward, partial topography-guided surface abla- tion laser treatment was applied. Topography-guided custom ablation treatment was performed by linking the Topolyzer with the WaveLight Allegretto Wave ${ }^{\mathrm{TM}}$ Excimer Laser System (Erlanger, Germany). In order to remove the minimum possible tissue, the optical zone diameter is kept between 5.5 and $6.5 \mathrm{~mm}$ in all cases. Maximum central ablation depth was 20 to $50 \mu \mathrm{m}$. The aim of this procedure was to topographically neutralize the cornea, making it more regular, and not necessarily a reduction in the spherical equivalent of the eye. A cellulose sponge soaked in mitomycin C $0.02 \%$ solution was applied over the ablated tissue for 30 seconds followed by irrigation with $20 \mathrm{~mL}$ of chilled balanced salt solution.

Step 2: Collagen cross-linking procedure

Riboflavin $(0.1 \%$ solution $10 \mathrm{mg}$ riboflavin-5-phosphate in $10 \mathrm{~mL}$ dextran-T-500 20\% solution) was applied every 2 minutes for approximately 20 minutes until the stroma was completely penetrated and the aqueous was stained yellow (riboflavin shielding). A commercially available UVA system (INOC) was used for UVA (365 nm) irradiation. Before treatment, the intended $9 \mathrm{~mW} / \mathrm{cm}^{2}$ surface irradiance $\left(5.4 \mathrm{~J} / \mathrm{cm}^{2}\right.$ surface dose after 10 minutes) was calibrated. During treatment, riboflavin solution was applied every 2 minutes to ensure saturation. After treatment, a bandage contact lens was applied until the epithelium healed completely. The patients were put on a tapering dose of prednisolone acetate $1 \%$ eye drops, topical antibiotic, and a lubricating eye drop.

Patients were followed up on day 1 , day 7 , and then at 1, 3, 6, and 12 months. Uncorrected visual acuity, BCVA, manifest refraction, cycloplegic refraction, slit-lamp examination of the anterior and posterior segments of the eye, keratometry readings, pachymetry, and corneal topography were performed at each visit.

\section{CASE REPORTS}

\section{Case 1}

A 37-year-old male presented to us with keratoconus in his right eye, intolerant to spectacles and contact lens. His right eye UCVA was $6 / 600$ and BCVA $6 / 15$ with a spectacle correction of $+3.0 /-4.0^{*} 60$. He underwent T-CAT with CXL for his right eye. Topography-guided custom ablation treatment was performed with a refractive correction of $-1.00 /-6.30 @ 11^{\circ}$, maintaining the central ablation to be $72.5 \mu \mathrm{m}$.

Postoperatively, his UCVA was $6 / 24$ in the right eye and BCVA was 6/10 with a spectacle correction of -2.00/-4.0 DC@8 ${ }^{\circ}$. His Difference Map (Fig. 1) reveals flattening of the inferior steep cone and steepening of the 

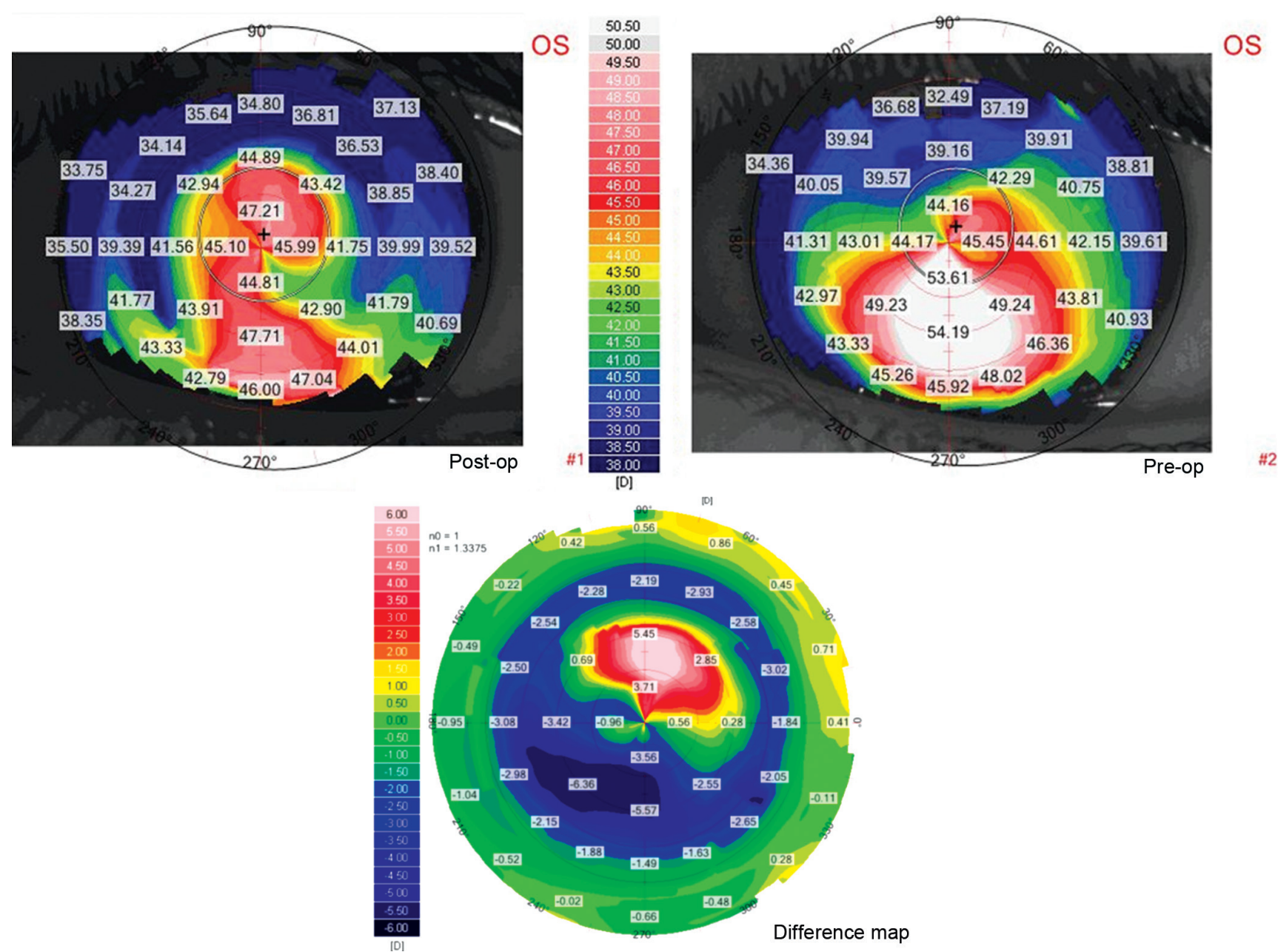

Fig. 1: RE Case 1 - Difference Map - preoperative map showing inferior steepening. Post-TCAT map showing flattening of the inferior steep cone and steepening the superior cornea

superior cornea. His refraction and tomography are kept stable on follow-up visits for the last 8 months.

\section{Case 2}

A 24-year-old female presented to us with keratoconus in her right eye. Her right eye UCVA was 6/30, and BCVA was $6 / 12$ with a spectacle correction of $+0.0 /-6.0 @ 35^{\circ}$. Her preoperative Scheimpflug map shows asymmetric bow-tie with inferior steepening, suggesting keratoconus.

She underwent T-CAT + surface ablation with CXL for her right eye. Topography-guided custom ablation treatment was performed with a refractive correction of $-0.3 /-5.0 @ 30$, maintaining the central ablation to be 76 microns.

Postoperatively, her UCVA was 6/15 in the right eye and BCVA was 6/9 with a spectacle correction of $+0.25 /-1.75 @ 20^{\circ}$. Her Difference Map (Fig. 2) reveals flattening of the inferior steep cone and steepening the superior cornea. Her refraction and tomography are kept stable on follow-up visits for the last 2.5 years.

\section{Case 3}

A 14-year-old male presented to us with keratoconus in both eyes. His right eye UCVA was 6/60, and BCVA was $6 / 18$ with a spectacle correction of $-2.25 /-6.5^{*} 5$. His left eye UCVA was $6 / 60$, and BCVA was $6 / 12$ with a spectacle correction of $-1.25 /-6.25 @ 160^{\circ}$.

He underwent T-CAT + surface ablation with CXL for both eyes. Topography-guided custom ablation treatment was performed for his right eye with a refractive correction of $-1.25 /-7.40 @ 3^{\circ}$, maintaining the central ablation to be $72 \mu \mathrm{m}$.

Topography-guided custom ablation treatment was performed for his left eye with a refractive correction of $-0.13 /-5.63 @ 160^{\circ}$, maintaining the central ablation to be $75 \mu \mathrm{m}$.

Postoperatively, his UCVA was 6/30 in the right eye and 6/9 in the left eye, and BCVA was $6 / 9$ with a spectacle correction of $-3.75 /-2.250 @ 20^{\circ}$ in the right eye and 6/7.5 with a spectacle correction of $-1.25 /-2.25 @ 150^{\circ}$ in the left eye. His Difference Map in the left eye (Fig. 3) revealed regularization of the cornea with flattening of the $\mathrm{K}$ values 

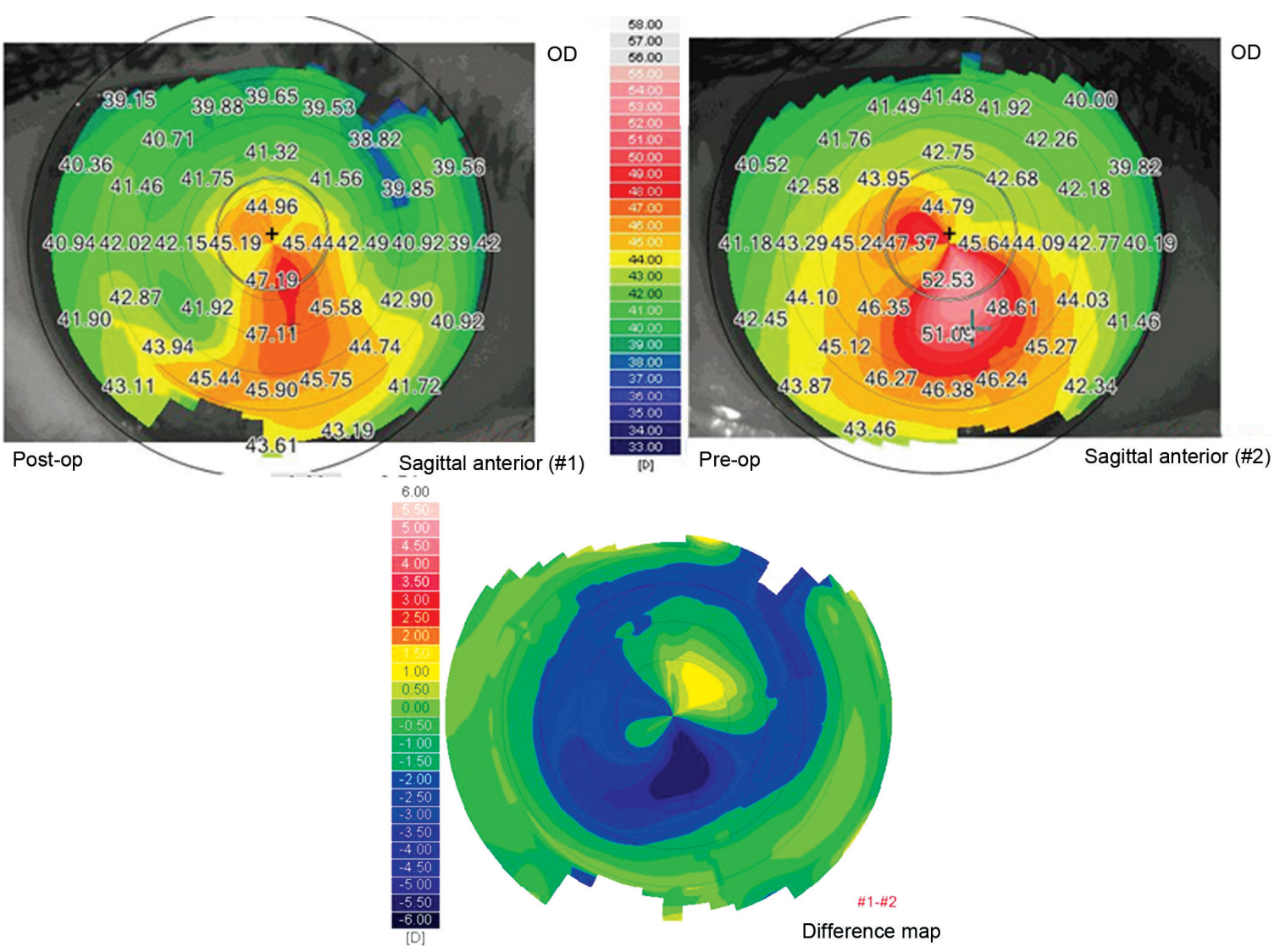

Fig. 2: RE Case 2 - Difference Map - preoperative map showing asymmetric bow-tie with inferior steepening. Post-TCAT map showing flattening of the $\mathrm{K}$ values
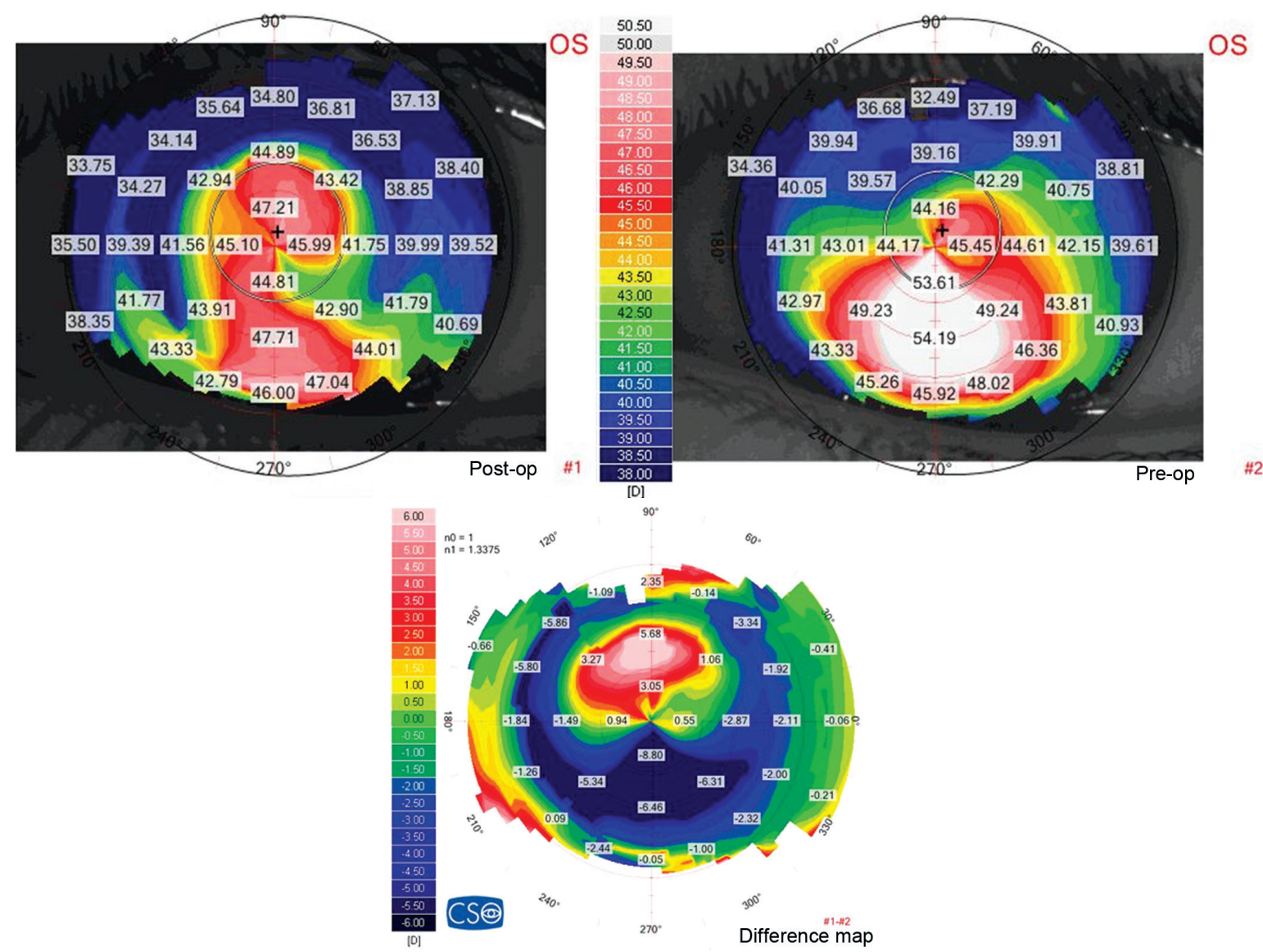

Fig. 3: LE Case 3 - Difference Map - preoperative map showing asymmetric bow-tie with inferior steepening. Post-TCAT map showing flattening of the $\mathrm{K}$ values 
Simultaneous Topography-guided Surface Ablation with Collagen Cross-linking for Keratoconus

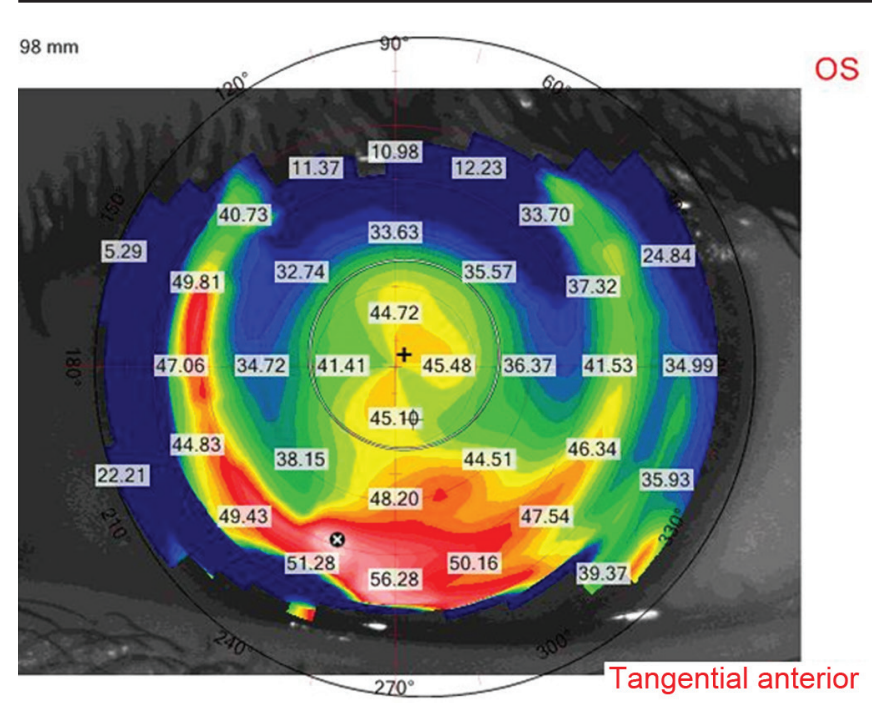

Fig. 4: LE Case 3 - Tangential Map -2 years after TCAT showing that his tomography is kept stable

at various points. His refraction and tomography are kept stable on follow-up visits for the last 2 years (Fig. 4).

\section{RESULTS}

Table 1 presents a summary of eight cases who were treated with TCAT+CXL for keratoconus. All eyes experienced improvement in BCVA.

Most patients complained of pain on the first postoperative day, whereas others reported minimal discomfort. There was no significant change in the intraocular pressure, no problems related to epithelial healing, no cases of stromal scarring, and no adverse events.

\section{DISCUSSION}

Simultaneous T-CAT surface ablation with CXL offers keratoconic patients intolerant to contact lenses both stabilization of the cornea and improved functional vision with spectacles correction. Reaching functional vision consists of improving UCVA and BCVA, and normalization of corneal topography, making these patients less dependent on contact lenses to achieve higher postoperative vision quality.

The combination of T-CAT and CXL has been described earlier as a two-step procedure with CXL being done first, followed by surface ablation after a 1-year interval, as well as a simultaneous procedure.

Same-day simultaneous topography-guided surface ablation and CXL has several advantages over the sequential technique ${ }^{10}$ :

- The combination reduces the patient's time away from work

- Performing both procedures at the same time with topography-guided surface ablation appears to minimize the potential superficial stromal scarring and haze resulting from topography-guided surface ablation. ${ }^{11}$

- When topography-guided PRK is performed following the CXL procedure, some of the cross-linked anterior cornea is removed, minimizing the potential benefit of CXL.

- By removing the Bowman layer with topographyguided surface ablation may facilitate riboflavin solution penetration in the corneal stroma and less "shielding" of UVA light in its passage through the cornea, resulting in more effective CXL.

- More predictable refractive results and visual outcome due to ablation of normal noncross-linked tissue.

The ablation is capable of reshaping the corneal surface, and CXL then halts progression of the disorder.

Table 1: Central ablation depth, pre- and postoperative UCVA and BCVA, and safety and efficacy indexes in 8 T-CAT-surface ablation + CXL-treated eyes

\begin{tabular}{|c|c|c|c|c|c|c|c|c|}
\hline Case numbers & Case type & UCVA & $B C V A$ & Subjective refraction & $\begin{array}{l}\text { Central ablation } \\
\text { depth }\end{array}$ & $\begin{array}{l}\text { Efficacy } \\
\text { index }\end{array}$ & $\begin{array}{l}\text { Safety } \\
\text { index }\end{array}$ & $\begin{array}{l}\text { Follow-up } \\
\text { time (months) }\end{array}$ \\
\hline \multirow[t]{2}{*}{$1-\mathrm{RE}$} & Preoperative & $6 / 600$ & $6 / 15$ & $+3.0 /-4.0 * 60$ & 40 & 0.625 & 1.5 & 8 \\
\hline & Postoperative & $6 / 24$ & $6 / 10$ & $-2.0 /-4.0 * 8$ & & & & \\
\hline \multirow[t]{2}{*}{$2-\mathrm{RE}$} & Preoperative & $6 / 30$ & $6 / 12$ & $0.0 /-6.0 * 35$ & 62 & 0.8 & 1.33 & 29 \\
\hline & Postoperative & $6 / 15$ & $6 / 9$ & $+0.25 /-1.75^{\star} 20$ & & & & \\
\hline \multirow[t]{2}{*}{$3-\mathrm{RE}$} & Preoperative & $6 / 60$ & $6 / 18$ & $-2.25 /-6.5^{\star} 5$ & 33 & 0.6 & 2 & 23 \\
\hline & Postoperative & $6 / 30$ & $6 / 9$ & $-3.75 /-2.25^{\star} 20$ & & & & \\
\hline \multirow[t]{2}{*}{$3-\mathrm{LE}$} & Preoperative & $6 / 60$ & $6 / 12$ & $-1.25 /-6.25 * 160$ & 39 & 1.33 & 1.6 & 21 \\
\hline & Postoperative & $6 / 9$ & $6 / 7.5$ & $-1.25 /-2.25 * 150$ & & & & \\
\hline \multirow[t]{2}{*}{$4-\mathrm{RE}$} & Preoperative & $6 / 60$ & $6 / 12$ & $+1.25 /-6.5^{\star} 58$ & 20 & 0.4 & 1.2 & 2 \\
\hline & Postoperative & $6 / 30$ & $6 / 10$ & $-2.75 /-1.25^{\star} 15$ & & & & \\
\hline \multirow[t]{2}{*}{4 - LE } & Preoperative & $6 / 60$ & $6 / 20$ & $-2.75 /-7.0 * 180$ & 49 & 0.83 & 1.66 & 11 \\
\hline & Postoperative & $6 / 24$ & $6 / 12$ & $+1.25 /-4.0 * 180$ & & & & \\
\hline \multirow[t]{2}{*}{$5-\mathrm{RE}$} & Preoperative & $6 / 60$ & $6 / 30$ & $+2.0 /-3.0 * 30$ & 58 & 1.25 & 2 & 3 \\
\hline & Postoperative & $6 / 24$ & $6 / 15$ & $+1.50 /-1.5^{*} 80$ & & & & \\
\hline \multirow[t]{2}{*}{$5-\mathrm{LE}$} & Preoperative & $6 / 20$ & $6 / 15$ & $+1.0 /-3.0 * 120$ & 50 & 1.6 & 2 & 3 \\
\hline & Postoperative & $6 / 9$ & $6 / 7.5$ & $+0.25 /-1.75^{\star} 80$ & & & & \\
\hline
\end{tabular}


The general changes produced by this combined treatment modality are flattening of the cone and improvement of the overall shape. This occurs because this treatment flattens the inferior steepest area and steepens the superior flattest area. After the procedure, patients are easier to fit with glasses or contact lenses, which are also better tolerated than before. Increased functionality and delayed or eliminated need for penetrating keratoplasty are also benefits of this treatment.

In this article, we report our results of this combined procedure. There was a rapid and significant improvement in UCVA and BCVA in 100\% eyes, a reduction of keratometric values, and symmetry between vertical hemimeridians. Topographic evaluation showed a marked improvement in irregularity.

There were no signs of keratoconic progression noted in any of the eyes on last follow-up. No adverse events were reported in any patient.

Our findings correspond with results reported by Kanellopoulos ${ }^{7}$ with same-day, simultaneous topographyguided surface ablation and CXL as a therapeutic intervention in highly irregular corneas with progressive corneal ectasia. Simultaneous surface ablation followed by CXL seems to be a promising treatment capable of offering patients functional vision and halting progression of the disorder.

Larger, comparative studies establishing the safety and efficacy of this treatment and longer follow-up are necessary in order to further validate these results and potentially make this treatment available for ectatic corneal disorders.

\section{REFERENCES}

1. Rabinowitz YS. Keratoconus. Surv Ophthalmol 1998 JanFeb;42(4):297-319.

2. Siganos CS, Kymionis GD, Kartakis N, Theodorakis MA, Astyrakakis N, Pallikaris IG. Management of keratoconus with Intacs. Am J Ophthalmol 2003 Jan;135(1):64-70.

3. Koller T, SchumacherS, Fankhauser F 2nd, Seiler T. Riboflavin/ ultraviolet a crosslinking of the paracentral cornea. Cornea 2013 Feb;32(2):165-168.

4. Koller T, Pajic B, Vinciguerra P, Seiler T. Flattening of the cornea after collagen crosslinking for keratoconus. J Cataract Refract Surg 2011 Aug;37(8):1488-1492.

5. Hafezi F, Koller T, Vinciguerra P, Seiler T. Marked remodelling of the anterior corneal surface following collagen cross-linking with riboflavin and UVA. Br J Ophthalmol 2011 Aug;95(8):1171-1172.

6. Kanellopoulos AJ, Binder PS. Management of corneal ectasia after LASIK with combined, same-day, topography-guided partial transepithelial PRK and collagen cross-linking: the athens protocol. J Refract Surg 2011 May;27(5):323-331.

7. Kanellopoulos AJ. Comparison of sequential vs same-day simultaneous collagen cross-linking and topography-guided PRK for treatment of keratoconus. J Refract Surg 2009 Sep;25(9):S812-S818.

8. Kanellopoulos AJ, Binder PS. Collagen cross-linking (CCL) with sequential topography-guided PRK: a temporizing alternative for keratoconus to penetrating keratoplasty. Cornea 2007 Aug;26(7):891-895.

9. Koller T, Iseli HP, Donitzky C, Ing D, Papadopoulos N, Seiler T. Topography-guided surface ablation for forme fruste keratoconus. Ophthalmology 2006 Dec;113(12):2198-2202.

10. Krueger RR, Kanellopoulos AJ. Stability of simultaneous topography-guided photorefractive keratectomy and riboflavin/UVA cross-linking for progressive keratoconus: case reports. J Refract Surg 2010 Oct;26(10):S827-S832.

11. Tuwairqi WS, Sinjab MM. Safety and efficacy of simultaneous corneal collagen cross-linking with topography-guided PRK in managing low-grade keratoconus: 1-year follow-up. J Refract Surg 2012 May;28(5):341-345. 